\title{
Dictynna
}

Dictynna

Revue de poétique latine

$3 \mid 2006$

Varia

\section{Padri e figlie: suggestioni ovidiane in una novella di Boccaccio (Decameron 5, 7)}

\section{Mario Labate}

\section{OpenEdition}

\author{
Journals
}

Edizione digitale

URL: http://journals.openedition.org/dictynna/218

DOI: $10.4000 /$ dictynna.218

ISSN: 1765-3142

Notizia bibliografica digitale

Mario Labate, «Padri e figlie: suggestioni ovidiane in una novella di Boccaccio (Decameron 5,7$)$ », Dictynna [En ligne], 3 | 2006, mis en ligne le 30 novembre 2010, consulté le 10 septembre 2020. URL http://journals.openedition.org/dictynna/218; DOI : https://doi.org/10.4000/dictynna.218

Questo documento è stato generato automaticamente il 10 settembre 2020

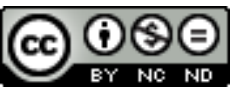

Les contenus des la revue Dictynna sont mis à disposition selon les termes de la Licence Creative Commons Attribution - Pas d'Utilisation Commerciale - Pas de Modification 4.0 International. 


\title{
Padri e figlie: suggestioni ovidiane in una novella di Boccaccio (Decameron 5, 7)
}

\author{
Mario Labate
}

1 "Boccaccio medievale", titolo di un libro famoso di uno dei maestri della filologia moderna, ${ }^{1}$ è anche una formula critica efficace, di quelle che orientano immediatamente chi si accosti al problema delle coordinate culturali e letterarie entro cui collocare la narrativa boccacciana.

2 Al di là delle intenzioni provocatorie che deliberatamente assunse nel quadro della critica boccacciana del suo tempo, la fortunata formula di Branca non nasceva da smanie definitorie, dal bisogno di etichette nuove che rimediassero, per via di bilanciamento, alla separazione del Decameron dal mondo ideologico di San Tommaso e di Dante o all'effetto che poteva avere, nella valutazione complessiva dell'autore, un'attenzione unilaterale per il Boccaccio erudito, protoumanista, inserito in una tradizione "classico-rinascimentale": nasceva piuttosto da un lungo e paziente lavoro di indagine filologica sui testi e le tradizioni di cui Boccaccio narratore propone una originale rielaborazione e sintesi.

3 Premetto che non è mia intenzione, quand'anche agisse in me un'inconscio patriottismo di antichista e di classicista, mettere in discussione i presupposti e la complessiva validità di questo classico degli studi. E' tuttavia mia convinzione che tutti i fondamenti di una cultura critica dovrebbero di tanto in tanto essere sottoposti a verifica, non solo per saggiarne la tenuta alla luce di prospettive nuove e consapevolezze diverse, ma anche per contrastare il naturale processo di sclerotizzazione che porta una buona idea a tradursi in vulgata, e finisce per dare opacità ai testi e a impedire oggettivamente quelle nuove pazienti indagini filologiche, quel lavoro di scavo che è la base indispensabile di ogni rinnovamento.

4 Per 'tastare il polso' alla situazione attuale delle conoscenze, è probabilmente utile rivolgersi, più ancora che alla saggistica e agli studi speciali, all'assetto secondo cui ogni 
singolo problema viene sistemato nelle opere manualistiche, nei grandi strumenti di consultazione, nei dizionari.

Vediamo ad esempio, dello stesso Branca, la voce Boccaccio nel Dizionario Critico della Letteratura Italiana: ${ }^{2}$

Gli argomenti derivano molto raramente dalle letterature classiche e più spesso da narrazioni dotte o popolaresche dei secoli immediatamente precedenti

Nella materia quanto mai varia e complessa del Decameron il mondo classico è poco meno che assente.

Le uniche due novelle per cui si possa richiamare una fonte classica, cioè la decima della $\mathrm{V}$ giornata e la seconda della VII giornata, non prescindono da intermediari e contaminazioni con versioni posteriori testimoniate da fabliaux e da racconti popolari

Egli dalle sue vaste conoscenze delle letterature classiche avrebbe potuto, più di ogni altro suo contemporaneo, trarre ampia materia di narrazione: e ne trasse, difatti, per le sue opere successive, come in altri suoi scritti precedenti...aveva riecheggiato, sia pure esteriormente, quegli alti modelli. Nel Decameron, al contrario, anche quando gli si offrono naturali e suggestivi i modelli classici, il B. sembra deliberatamente sfuggirli ed escluderli, per rivolgersi ad ammirati testi medievali.

Un'altra acquisizione della critica boccacciana è la consapevolezza che la narrazione del Decameron nasce da un raffinato lavoro di riscrittura:

"Boccaccio mette generalmente in atto un complicatissimo gioco di contaminazione (e/o parodia) di modelli, motivi, lacerti testuali di eterogenea provenienza, provocando un singolare, inevitabilmente straniante, effetto di 'sovraimpressione'. Per quanto consentono di verificare i dati a nostra conoscenza e le ricerche fin qui compiute, la stragrande maggioranza delle novelle decameroniane nasce da questa abilissima strategia di bricolage". ${ }^{3}$

7 Scopo di questo mio intervento è mostrare, attraverso un esempio, come, nel quadro dei materiali letterari e narrativi che svolgono una funzione rilevante nel laboratorio dell'intertestualità decameroniana, la presenza della letteratura classica sia meno modesta di quanto sembrerebbe risultare dalla communis opinio prima ricordata, e non si eserciti soltanto in maniera indiretta, o al livello di lontane matrici culturali o di archetipi di generi, modalità o registri narrativi, ma possa anche proporsi come specifico rapporto tra testi. L'autore classico è Ovidio, il testo una delle Heroides (precisamente la XI, l'epistola di Canace a Macareo), la novella del Decameron in cui questa epistola ovidiana agisce, secondo me, in filigrana come principale ipotesto è la settima della quinta giornata ("Teodoro, innamorato della Violante, figliuola di messere Amerigo suo signore, la 'ngravida e è alle forche condannato; alle quali frustandosi essendo menato, dal padre riconosciuto e prosciolto prende per moglie la Violante").

Per argomentare quanto Ovidio sia un ottimo candidato a incrementare il ristretto numero degli autori antichi che si può pensare attivamente presenti nella scrittura decameroniana non c'è bisogno di spendere molte parole. Basterà ricordare il ruolo centrale che, nella poetica di Boccaccio narratore, svolge il magister amoris, in particolare l'Ovidio dei Remedia, che impartisce la terapia della passione infelice e l'antidoto ai suoi esiti tragici: lo hanno acutamente indagato specialisti come Luciano Rossi e Robert Hollander. ${ }^{4}$

Quanto poi alle Heroides, lo sguardo da un punto di vista femminile sulle vicende d'amore è stato riconosciuto come uno dei tratti più innovativi e originali di questo esperimento letterario ovidiano ${ }^{5}$ : la suggestione esercitata specificamente dalle Heroides 
sulla poetica del Decameron, come opera che individua appunto nelle donne il suo orizzonte di pubblico, è dimostrata dalla nota allusione del proemio stesso all'epistola di Ero a Leandro, nella descrizione dei limiti che chiudono la vita delle donne in uno spazio angusto e monotono ${ }^{6}$, all'interno del quale le sofferenze d'amore si comprimono, si dilatano e rischiano di deflagrare nella tragedia:

Bocc. Decam. proem. 9-12

E chi negherà questo, quantunque egli si sia, non molto più alle vaghe donne che agli uomini convenirsi donare? Esse dentro a' dilicati petti, temendo e vergognando, tengono l'amorose fiamme nascose, le quali quanto più di forza abbian che le palesi coloro il sanno che l'hanno provate: e oltre a ciò, ristrette da' voleri, da' piaceri, da' comandamenti de' padri, delle madri, de' fratelli e de' mariti, il più del tempo nel piccolo circuito delle loro camere racchiuse dimorano e quasi oziose sedendosi, volendo e non volendo in una medesima ora, seco rivolgendo diversi pensieri, li quali non è possibile che sempre sieno allegri. $\mathrm{E}$ se per quegli alcuna malinconia, mossa da focoso disio, sopraviene nelle lor menti, in quelle conviene che con grave noia si dimori, se da nuovi ragionamenti non è rimossa: senza che elle sono molto men forti che gli uomini a sostenere; il che degli innamorati uomini non avviene, sì come noi possiamo apertamente vedere. Essi, se alcuna malinconia o gravezza di pensieri gli affligge, hanno molti modi da alleggiare o da passar quello, per ciò che a loro, volendo essi, non manca l'andare a torno, udire e veder molte cose, uccellare, cacciare, pescare, cavalcare, giucare o mercatare: de' quali modi ciascuno ha forza di trarre, o in tutto o in parte, l'animo a sé e dal noioso pensiero rimuoverlo almeno per alcuno spazio di tempo, appresso il quale, con un modo o con altro, o consolazion sopraviene o diventa la noia minore.

Ov. Her. 19, 5-16

urimur igne pari, sed sum tibi viribus inpar:

fortius ingenium suspicor esse viris.

ut corpus, teneris ita mens infirma puellis:

deficiam, parvi temporis adde moram!

vos modo venando, modo rus geniale colendo

ponitis in varia tempora longa mora.

aut fora vos retinent aut unctae dona palaestrae,

flectitis aut freno colla sequacis equi;

nunc volucrem laqueo, nunc piscem ducitis hamo;

diluitur posito serior hora mero.

his mihi summotae, vel si minus acriter urar,

quod faciam, superest praeter amare nihil.

10 Nella quinta giornata del Decameron, che è posta sotto il reggimento di Fiammetta, "si ragiona di ciò che a alcuno amante, dopo alcuni fieri o sventurati accidenti, felicemente avvenisse".

11 Ripercorriamo in breve la trama della novella raccontata, all'interno di questo programma narrativo, da Lauretta $(5,7)$ :

12 Siamo in Sicilia, a Trapani, al tempo di Guglielmo il Buono. Un ricco gentiluomo, Amerigo Abate, compra, per il servizio della sua casa, giovani schiavi che dei corsari genovesi avevano razziato in Armenia: tra questi, un fanciullo di nome Teodoro, che si distingue per nobiltà d'aspetto e di carattere. Il ragazzo cresce in casa di Amerigo accanto ai suoi figli, viene convenientemente educato e diventa il beniamino del padrone, che lo affranca, lo fa battezzare col nome di Pietro e ne fa il proprio uomo di fiducia. Violante, figliola di Amerigo, che il padre tarda a maritare, si innamora del giovane e ne viene ricambiata, ma il pudore impedisce ai due giovani di rivelare $\mathrm{i}$ reciproci sentimenti, finché a sbloccare la situazione non interviene la fortuna: le donne di casa, accompagnate da Pietro, si recano in una proprietà suburbana e, al 
ritorno, allo scoppio di un violento temporale, Violante e Pietro, isolati dal resto della compagnia, trovano riparo in una chiesetta diroccata, dove finalmente si rivelano l'un l'altro e consumano appassionatamente il loro amore. La successiva consuetudine amorosa non resta senza conseguenze e Violante rimane gravida. I due giovani sono disperati e, falliti i tentativi di interrompere la gravidanza, Pietro pensa di fuggire per sottrarsi all'ira di Amerigo, che egli immagina disposto al perdono nei confronti della figlia, ma implacabile nei suoi confronti. Violante lo convince a restare promettendo di tenere il segreto sulle sue responsabilità. Non potendo più celare il suo stato, Violante ne fa partecipe la madre, la quale, pur addolorata, cerca di salvarla e di nascondere il suo peccato, facendola trasferire in una proprietà di campagna. Ma le precauzioni materne sono vanificate dalla fortuna: Amerigo, contro le sue abitudini, si reca a cacciare in quella sua proprietà, proprio quando la figlia è giunta al termine della gravidanza: i dolori del parto fanno gridare la giovane e rivelano al padre la situazione. Amerigo non è disposto a credere che Violante ignori chi sia il padre del bambino e, minacciando con la spada la figlia, ne ottiene una piena confessione. Appreso del tradimento di Pietro, Amerigo, fieramente sdegnato, pretende da Currado, capitano della città di Trapani, che Pietro sia preso, torturato e mandato a morte come reo confesso. Da parte sua, Amerigo, non soddisfatto nella sua ira, incarica un suo famigliare di recare a Violante del veleno e un coltello (sarà lei a scegliere come darsi la morte) e di uccidere il neonato e darlo in pasto ai cani. Mentre Pietro è trascinato al patibolo viene notato da Fineo, un nobile armeno che, membro di un'importante ambasceria diretta a Roma per trattare col papa della crociata, si trova a far tappa a Trapani. La macchia vermiglia che si nota sul petto del condannato gli fa ricordare di un suo figlio rapito quindici anni primi e un breve interrogatorio del giovane conferma il suo sospetto. A questo punto, la macchina della fortuna comincia rapidamente a girare in senso inverso: Fineo si precipita da Currado e ottiene subito la sospensione della pena; Amerigo, cui viene rivelata l'identità di Pietro-Teodoro e vengono offerte nozze riparatrici, si pente della sua precipitosa crudeltà; un messo, immediatamente inviato, trova che, per fortuna, il duplice comando di morte non è stato ancora eseguito. Nessun ostacolo dunque per il lieto fine: Teodoro prima e Violante poi accettano con entusiasmo di unirsi in matrimonio. Tornato Fineo da Roma, le nozze vengono celebrate e i giovani sposi con il bambino tornano infine in Armenia, dove trascorrono una vita felice e tranquilla.

Dai repertori di fonti e dai commenti non risulterebbe che in questa novella, al di là di singoli limitati spunti e di un generico riferimento alla tradizione romanzesca, siano mobilitate importanti risorse di carattere intertestuale:

"There is nothing to say as to the source of this tale"

"Nessun antecedente per questa novella (il Gröber ${ }^{8}$ pensa, come al solito in questi casi, a ipotetici romanzi greci)...Un qualche presentimento della scena centrale (9-17) si potrebbe se mai scorgere nei versi virgiliani - ammiratissimi dal B. e da lui puntualmente imitati nell'Amorosa Visione (XXVIII) - sul temporale pronubo degli amori di Didone ed Enea (Aen., IV 160 ss.)".?

Io credo, invece, che il racconto di questa novella trovi il suo senso narrativo, e la sua coerenza al tema della giornata, attraverso una trama abbastanza fitta di riferimenti intertestuali, in cui il ruolo centrale è esercitato dalla epistola ovidiana di Canace a Macareo. E' l'intertesto ovidiano che, a un certo punto, sembra imporsi sull'iniziale spunto romanzesco, orientando decisamente (anche se momentaneamente) la storia dei due giovani amanti verso una cupa tragedia, attraverso il contrasto violento tra un 
padre autoritario e irascibile e la sua fragile figlia, vinta da una passione d'amore che le circostanze hanno reso irresistibile. Quando ormai la falsariga del modello ovidiano appare destinata a condurre i protagonisti verso un destino di morte, l'improvviso riaffiorare del tema romanzesco del fanciullo rapito e del gioco della fortuna conduce all'anagnorisis e a quell'insperato lieto fine che la regina ha imposto ai narratori come motivo guida della giornata.

L'undicesima epistola delle Heroides ricava la sua finestra narrativa su un mito tragico, quello dell'amore colpevole e incestuoso fra i due figli del re dei venti, Eolo, dal cui nome significativamente si intitolava la tragedia euripidea che ne costituisce l'antefatto' letterario. ${ }^{10}$ Canace scrive quando la sua storia sembra essersi consumata: terminata la lettera, non le resterà che usare contro sé stessa la spada ch'ella regge con la mano sinistra, mentre la destra sta vergando il suo disperato addio al fratelloamante:

Ov. Her. 11. 0a-4

Aeolis Aeolidae, quam non habet ipsa, salutem mittit et armata verba notata manu.

Siqua tamen caecis errabunt scripta lituris, oblitus a dominae caede libellus erit.

dextra tenet calamum, strictum tenet altera ferrum, et iacet in gremio charta soluta meo.

16 La trama dell'Aeolus di Euripide ci è un po'meglio nota da quando un papiro di Ossirinco (POxy 2457) ha restituito l'hypothesis della tragedia, le cui informazioni si aggiungono alle fonti mitografiche e a quel poco che, sullo sviluppo dell'azione drammatica, si può ricavare dai frammenti. ${ }^{11}$ Eolo, l'omerico re dei venti, ha nella sua casa sei figli e sei figlie: il suo figlio più giovane, Macareo, seduce la sorella Canace, che rimane incinta, ma tiene nascosta la sua gravidanza. Macareo riesce a convincere il padre dell'opportunità di far sposare i fratelli alle sorelle, ma il sorteggio con cui il padre decide di formare le coppie ha esito sfavorevole per Macareo: Canace viene assegnata a un altro. A questo punto, la situazione precipita: il bambino è nato e, nonostante l'aiuto della nutrice, la colpa di Canace viene scoperta da Eolo, che manda alla figlia una spada. Intepretando il comando di morte, Canace si uccide. Macareo, che nel frattempo è riuscito a placare l'ira paterna, corre da lei, ma, trovandola già morta, si uccide a sua volta con la stessa spada.

17 La tragedia euripidea si sviluppava probabilmente attorno a due nuclei drammatici: nella prima parte, il contrasto tra l'altezzosa regalità di Eolo e le argomentazioni di carattere morale ed etico-politico con cui Macareo cercava di convincerlo alle sue ragioni, nella discussione sul matrimonio; nella seconda parte (sul cui sviluppo drammatico siamo meno certi per l'interrompersi dell'hypothesis), il contrasto tra il violento autoritarismo del padre e la indifesa debolezza della figlia e del suo bambino, ambedue spezzati da una implacabile ira, impermeabile alle istanze degli affetti e della pietà. Sulla base di questo nucleo drammatico euripideo, secondo la logica di un genere che piega la voce sofferente dell'elegia a interpretare il punto di vista di una femminilità debole, esposta alla indifferenza, alla insensibilità al cinismo e anche alla brutalità degli uomini, Ovidio costruiva il personaggio della sua eroina: in punto di morte, Canace denunciava, con rassegnata e sommessa amarezza, la fine delle sue speranze, il suo dolore di madre impotente di fronte allo strazio della sua creatura e di figlia costretta a misurare la spietatezza di un padre orgoglioso e iracondo. 
La quinta giornata del Decameron ospita, come prima ricordavamo, storie il cui lieto fine rovescia, al di là di ogni ragionevole speranza, attese narrative di segno opposto. La novella di Teodoro e Violante è fra quelle che più efficacemente rispondono a questo programma, proprio perché costruisce il suo racconto caricandosi progressivamente di tensione drammatica attraverso l'evocazione di vicende e personaggi della grande letteratura del passato (gli auctores più prestigiosi della letteratura antica e l'auctor della letteratura volgare). L'amore unisce due giovani che sono presi nell'ingranaggio di un amore irresistibile, che li porta a trasgredire convenienze e doveri: Violante sacrifica verginità e obbedienza filiale, ledendo il diritto del padre di darla in sposa a suo arbitrio, Pietro manca al suo debito di riconoscenza e rispetto nei confronti di un padrone che l'aveva allevato ed educato, e lo aveva poi sottratto alla condizione servile per dargli fiducia e responsabilità. Ma la trasgressività di questa unione non è presentata come colpevole, non compromette la fondamentale innocenza dei due giovani protagonisti, perché nasce, quasi inevitabilmente, dall'effetto combinato di affinità elettive e di prolungata vicinanza, di cui, semmai, è proprio il padre a portare qualche responsabilità:

Bocc. Decam. 5, 7, 4 s.

...tra' quali [scil. i servi comprati da Amerigo], quantunque tutti gli altri paressero pastori, n'era uno il quale gentilesco e di migliore aspetto che alcuno altro pareva, e era chiamato Teodoro. Il quale, crescendo, come che egli a guisa di servo trattato fosse nella casa pur co' figliuoli di messere Amerigo si crebbe; e traendo più alla natura di lui che all'accidente, cominciò a essere costumato e di bella maniera;

5, 7, 6 Come gli altri figliuoli di messere Amerigo, così similmente crebbe una sua figliuola chiamata Violante, bella e dilicata giovane, la quale, sopratenendola il padre a maritare, s'innamorò per avventura di Pietro; e amandolo e faccendo de' suoi costumi e delle sue opere grande stima, pur si vergognava a discovrirgliele.

Violante e Pietro vivono tutta la fase del loro inespresso innamoramento prigionieri del pudore, della vergogna, delle mille esitazioni di chi si perita a manifestare un sentimento la cui illiceità intimidisce entrambi. E' solo la fortuna che allenta i freni inibitori e li spinge a superare la soglia del proibito, scaricandoli in buona misura da ogni più grave responsabilità:

Bocc. Decam. 5, 7, 9:

Ma mentre che essi così parimente nell'amorose fiamme accesi ardevano, la fortuna, come se diliberato avesse questo voler che fosse, loro trovò via da cacciare la temorosa paura che gl'impediva.

20 L'addensarsi improvviso del temporale, la gragnuola di grandine, i due che restano isolati dal resto della compagnia, la misteriosa sacralità del luogo in cui si riparano $\mathrm{e}$ finalmente consumano il loro amore: è già stato rilevato come questo tratto del racconto proietti sui due giovani la grande ombra di Didone ed Enea:

Verg. Aen. 4, 160-8

Interea magno misceri murmure caelum

incipit, insequitur commixta grandine nimbus,

et Tyrii comites passim et Troiana iuuentus

Dardaniusque nepos Veneris diuersa per agros

tecta metu petiere; ruunt de montibus amnes. speluncam Dido dux et Troianus eandem deueniunt. prima et Tellus et pronuba Iuno dant signum; fulsere ignes et conscius aether conubiis summoque ulularunt uertice Nymphae. 
Se l'unione di Violante e Pietro riduce a dimensione più umana e borghese il sublime di questa unione eroica e regale, cui lo scatenarsi degli elementi naturali (terra aria fuoco e acqua) fornisce una specie di grandioso rituale cosmico, ${ }^{12}$ resta nondimeno aperta la prospettiva di una storia che pare incamminarsi verso sviluppi di tragedia e di morte:

Verg. Aen. 4, $169 \mathrm{~s}$.

ille dies primus leti primusque malorum

causa fuit.

Questa prospettiva è confermata dall'altro nobile intertesto che traluce dietro il reciproco rivelarsi dell'amore tra due giovani i quali, dopo aver a lungo covato dentro di sé il fuoco di una passione nascosta, si trovano in una situazione di intimità e di contatto ravvicinato che li spinge irresistibilmente uno nelle braccia dell'altro:

Bocc. Decam. 5, 7, 13

Pietro e la giovane, non avendo più presto rifugio, se n'entrarono in una chiesetta antica e quasi tutta caduta, nella quale persona non dimorava, e in quella sotto un poco di tetto, che ancora rimaso v'era, si ristrinsono amenduni, e costrinsegli la necessità del poco coperto a toccarsi insieme, il qual toccamento fu cagione di rassicurare un poco gli animi a aprire gli amorosi disii.

Dante, Inf. 5, 118-120

Ma dimmi: al tempo de' dolci sospiri,

a che e come concedette amore

che conosceste i dubbiosi disiri.

Se l'evocazione del fantasma di Didone proiettava su Violante un destino di morte inferta di propria mano con l'arma donata da chi di quella stessa morte era autore e ispiratore (con le parole di Ovidio, Her. 7, 191 praebuit Aeneas et causam mortis et ensem;/ ipsa sua Dido concidit usa manu), questa allusione a Francesca e Paolo conferma l'aspettativa tragica che sui due giovani amanti incomba un destino di morte spietatamente inferta da chi si sente in diritto di punire la loro colpa:

Dante, Inf. 5, 100-07

Amor , ch'al cor gentil ratto s'apprende,

prese costui della bella persona

che mi fu tolta; e 'l modo ancor m'offende

...

Amor condusse noi ad una morte.

Caina attende chi a vita ci spense.

E' a questo punto che la storia di Violante 'incontra' vistosamente quella di Canace e se ne fa accompagnare per un consistente tratto della narrazione, fino sulla soglia stessa dell'esito infausto.

La confrontabilità della vicenda della novella con quella dell'epistola ovidiana ha una specie di premessa nel fatto che, in ambedue i casi, l'amore nasce in un contesto di vicinanza e di affinità. I due giovani vivono uno accanto all'altro, nella stessa casa, nella stessa famiglia: Canace e Macareo sono fratello e sorella, Teodoro-Pietro viene allevato ed educato insieme ai figli di messer Amerigo. ${ }^{13}$ Ma né Ovidio né Boccaccio insistono sul carattere trasgressivo o morboso di questo sentimento: l'amore sboccia con naturalezza, in un contesto di ingenuità, pudicizia, senso di vergogna. Violante "bella e dilicata", lasciata in lunga e imprudente consuetudine con un giovane "il quale gentilesco e di migliore aspetto che alcuno altro pareva", e che "traendo più alla natura di lui che all'accidente, cominciò a esser costumato e di bella maniera", non può che innamorarsene ed esserne riamata, senza che nessuno dei due indulga al sentimento e osi manifestarlo. Nel caso di Canace, addirittura vediamo l'amore per il fratello 
sbocciare nell'animo di una fanciulla inesperta come qualcosa di totalmente nuovo, che essa fatica a riconoscere o anche a immaginare, fino a quando la serie di inequivocabili sintomi della malattia d'amore non porta la nutrice a rivelarle la diagnosi, subito confermata da un accesso di rossore e da una manifestazione di pudicizia:

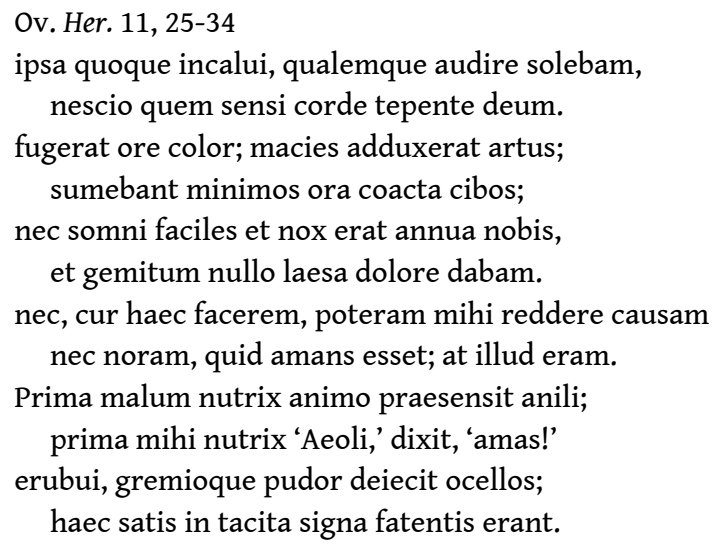

Come molti hanno riconosciuto, la Canace ovidiana non è la protagonista di un dramma della passione colpevole e morbosa, la cui forza travolgente trascina a violare regole e tabù, fino alla colpa dell'incesto affrontato a viso aperto, come sfida consapevole a tutto ciò che pone un limite alle pulsioni dell'eros: dalle Heroides emerge piuttosto una figura di giovane donna debole e ingenua, trascinata da una forza che non conosce e che non controlla, fino a subire la furia di un padre autoritario e spietato. ${ }^{14}$ Ciò che ha attratto Boccaccio verso l'epistola di Ovidio è la centralità in essa del rapporto padre-figlia, il contrasto tra la la fragilità femminile da una parte e la violenza di chi detiene l'autorità e ne esercita egoisticamente, in nome di un disumano senso dell'orgoglio e dell'onore, il potere repressivo.

Il contatto fra i due testi si fa evidente nelle conseguenze dell'inevitabile quanto sfortunato incontro dei due amanti (descritto nel Decameron attraverso le evocazioni virgiliana e dantesca di cui poc'anzi dicevamo, quasi del tutto censurato nelle Heroides, se non per reticente accenno della fanciulla stessa): ${ }^{15}$ una gravidanza indesiderata, cui seguono reiterati quanto inutili tentativi di abortire:

Ov. Her. 11, 37-44iamque tumescebant vitiati pondera ventris, aegraque furtivum membra gravabat onus.

quas mihi non herbas, quae non medicamina nutrix attulit audaci supposuitque manu,

ut penitus nostris -hoc te celavimus unumvisceribus crescens excuteretur onus?

a, nimium vivax admotis restitit infans artibus et tecto tutus ab hoste fuit!

Bocc. Decam. 5, 7, 17

e sì andò la bisogna che la giovane ingravidò, il che molto fu all'uno e all'altro discaro; per che ella molte arti usò per dovere contro al corso della natura disgravidare, né mai le poté venir fatto.

Di fronte alla prospettiva che il padre venga a conoscenza della colpa commessa, seguono momenti di vera disperazione: Pietro teme per la sua vita e progetta la fuga, Violante minaccia di togliersi la vita se Pietro l'abbandona; Canace, straziata dai dolori del parto, vede la morte davanti a sé, e non riesce più quasi a trattenere le grida che inevitabilmente la smaschererebbero. Ma poi sembra schiudersi una momentanea prospettiva di salvezza, che nasce dall'iniziativa di uno dei due giovani. A parti invertite, in Ovidio è Macareo a recarsi dalla sorella per rincuorarla e infonderle fiducia 
che il futuro riservi un buon finale per la loro storia; in Boccaccio è invece Violante a rassicurare Pietro del suo destino, promettendogli di saper tenere segreta l'identità del responsabile del suo stato:

Ov. Her. 11, 59-62

et mihi 'vive, soror, soror o carissima,' dixti;

'vive nec unius corpore perde duos!

spes bona det vires; fratri nam nupta futura es.

illius, de quo mater, et uxor eris.'

Bocc. Decam. 5, 7, 20

Al quale la giovane disse: "Pietro, il mio peccato si saprà bene, ma sii certo che il tuo, se tu nol dirai, non si saprà mai". Pietro allora disse: "Poi che tu così mi prometti, io starò: ma pensa d'osservarlomi".

In entrambe le storie, la fanciulla trova conforto e aiuto in una figura 'materna', che raccoglie le sue confidenze, la aiuta e fa del suo meglio per tenere nascosta al padre prima la gravidanza e poi il parto. In Ovidio, questa funzione narrativa di adiuvante è riempita dalla vecchia e affettuosa nutrice; in Boccaccio, dalla madre stessa di Violante:

Ov. Her. 11, $33 \mathrm{~s}$.

Prima malum nutrix animo praesensit anili; prima mihi nutrix 'Aeoli,' dixit, 'amas!'

Ov. Her. 11, $39 \mathrm{~s}$.

quas mihi non herbas, quae non medicamina nutrix

attulit...?

Ov. Her. 11, 49 s.

'quid,' ait, 'tua crimina prodis?'

oraque clamantis conscia pressit anus.

Ov. Her. 11, $67 \mathrm{~s}$.

frugibus infantem ramisque albentis olivae

et levibus vittis sedula celat anus

Bocc. Decam. 5, 7, 22

La giovane...con grandissimo pianto un dì il manifestò alla madre, lei per la sua salute pregando".

Bocc. Decam. 5, 7, 23

La donna la si credette, e per celare il difetto della figliuola a una lor possessione ne la mandò.

Bocc. Decam. 5, 7, 26

La donna s'ingegnò, in quanto poteva, di dover fare stare contento il marito a quello che ella aveva detto, ma ciò era niente.

Ma è nel confronto tra padre e figlia che la novella e l'epistola trovano il più vistoso $\mathrm{e}$ significativo punto di contatto intertestuale. L'evento che si era cercato in ogni modo di nascondere viene svelato ad Amerigo dalle grida della partoriente:

Bocc. Decam. 5, 7, 24

Quivi, sopravenuto il tempo del partorire, gridando la giovane come le donne fanno, non avvisandosi la madre di lei che quivi messer Amerigo, che quasi mai usato non era, dovesse venire, avvenne che, tornando egli da uccellare e passando lunghesso la camera dove la figliuola gridava, maravigliandosi, subitamente entrò dentro e domandò che questo fosse.

31 La medesima eventualità era minacciosamente prospettata nel racconto di Canace, ma era stata momentaneamente evitata, grazie al provvidenziale intervento della nutrice e alla forza d'animo della ragazza, che riesce miracolosamente a controllarsi e a inghiottire grida, dolore e lacrime:

Ov. Her. 11, 45-54

Iam noviens erat orta soror pulcherrima Phoebi, 
et nova luciferos Luna movebat equos.

nescia, quae faceret subitos mihi causa dolores,

et rudis ad partus et nova miles eram.

nec tenui vocem. 'quid,' ait, 'tua crimina prodis?'

oraque clamantis conscia pressit anus.

quid faciam infelix? gemitus dolor edere cogit,

sed timor et nutrix et pudor ipse vetant.

contineo gemitus elapsaque verba reprendo

et cogor lacrimas conbibere ipsa meas.

Ma, quando la salvezza sembrerebbe ormai a portata di mano (la nutrice è quasi riuscita a portar fuori dal palazzo di Eolo il bambino, nascondendolo fra gli apparecchi di un presunto rito religioso), è sempre un grido, il vagito incontrollabile del neonato, che smaschera l'inganno e fa precipitare la situazione (Ov. Her. 11, 67-72). Il re dei venti ha una reazione furibonda, fa rimbombare la reggia delle sue urla, si precipita quindi contro la figlia, investendola di ingiurie e gridandone a tutti il disonore, a stento trattenendosi dal passare dalle parole alla violenza fisica:

Ov. Her. 11, 73-80

eripit infantem mentitaque sacra revelat

Aeolus; insana regia voce sonat.

inruit et nostrum vulgat clamore pudorem,

et vix a misero continet ore manus.

Messer Amerigo non si dimostra meno iracondo e violento:

Bocc. Decam. 5, 7, 27-28

Egli, salito in furore, con la spada ignuda in mano sopra la figliuola corse, la quale mentre di lei il padre teneva in parole aveva un figliuol maschio partorito, e disse: "O tu manifesta di chi questo parto si generasse, o tu morrai senza indugio".

La giovane, la morte temendo, rotta la promessa fatta a Pietro, ciò che tra lui e lei stato era tutto aperse; il che udendo il cavaliere e fieramente divenuto fellone, appena d'ucciderla si trattenne; ma poi che quello che l'ira gli apparecchiava detto l'ebbe...

L'ira smisurata conduce Eolo a una decisione crudele, che non soltanto esclude ogni indulgenza per le debolezze della figlia, ma si dimostra tanto sorda alle ragioni degli affetti e ai legami del sangue, da condannare spietatamente a morte anche il frutto del peccato di Canace, quel bambino innocente, suo nipote, il cui orribile destino viene rappresentato con un pathos non privo di indignazione:

Ov. Her. 11, 83-90

Iamque dari parvum canibusque avibusque nepotem iusserat, in solis destituique locis.

vagitus dedit ille miser-sensisse putaresquaque suum poterat voce rogabat avum.

quid mihi tunc animi credis, germane, fuissenam potes ex animo colligere ipse tuo-

cum mea me coram silvas inimicus in altas viscera montanis ferret edenda lupis?

E' a partire da questa figura di padre crudele che Boccaccio costruisce il suo personaggio:

Bocc. Decam. 5, 7, 29-31

E essendo [Pietro] dopo alcun dì dal capitano condannato che per la terra frustato fosse e poi appiccato per la gola, acciò che una medesima ora togliesse di terra i due amanti e il loro figliuolo, messere Amerigo, al quale per avere a morte condotto Pietro non era l'ira uscita, mise veleno in un nappo con vino e quello diede a un suo 
famigliare e un coltello ignudo con esso, e disse: "Va con queste due cose alla Violante e sì le dì da parte mia che prestamente prenda qual vuole di queste due morti, o del veleno o del ferro: se non, che io nel cospetto di quanti cittadini ci ha la farò ardere sì come ella ha meritato; e fatto questo, piglierai il figliuolo pochi dì fa da lei partorito e, percossogli il capo al muro, il gitta a mangiare a' cani". Data dal fiero padre questa crudel sentenzia contro alla figliuola e al nepote, il famigliare, più a male che a ben disposto, andò via.

Messer Amerigo pretende soddisfazione per il suo orgoglio ferito, la sua vendetta sarà consumata con feroce determinazione, ed egli sembra pascersi sadicamente dell'immagine di una figlia che riceve dal proprio genitore quei terribili doni (la facoltà di scegliere il dono paterno da usare contro se stessa sembra perfino aggiungere, al comando di morte, una nota di irrisione) ${ }^{16}$. Ancora più violenta è la ferocia nei confronti del nipote: Eolo lo aveva fatto esporre, destinandone il corpo ad essere pasto di cani ed uccelli, mentre Amerigo dispone che lo strazio del cadavere sia preceduto da una morte ancor più violenta e raccapricciante.

Anche il personaggio del servitore, incaricato di eseguire la sentenza del padre e di portare alla figlia lo strumento con cui darsi la morte, sovrappone la storia di Violante alla storia ovidiana di Canace:

Ov. Her. 11, 93-96

Interea patrius vultu maerente satelles venit et indignos edidit ore sonos:

'Aeolus hunc ensem mittit tibi' -tradidit ensem-

'et iubet ex merito scire, quid iste velit.'

Ancora una volta, Boccaccio, rinunciando al motivo (non raro in questo genere di racconto) dell'esecutore impietosito di fronte all'inerme fragilità della vittima, incupisce ulteriormente le tinte: se infatti, in Ovidio, il satelles esegue vultu maerente il suo compito ingrato, il famigliare di Amerigo sembra invece un vero 'doppio' del suo padrone, di cui fino all'ultimo riproduce l'accanimento e la fellonìa:

Bocc. Decam. 5, 7, 45

Colui che andò, trovò il famigliare stato da messer Amerigo mandato, che, avendole il coltello e 'l veleno posto innanzi, perché ella così tosto non eleggeva, le diceva villania e volevala costrignere di pigliar l'uno".

Il lettore delle novelle della quarta giornata aveva conosciuto nella storia di Ghismonda o in quella di Lisabetta la tragedia di una femminilità brutalmente piagata dal crudele esercizio dell'autorità familiare. Nella diversa atmosfera della giornata governata da Fiammetta, il sollievo del lieto fine fa dipendere la sua efficacia liberatoria da un accumulo di tensioni narrative decisamente orientate in direzione opposta. L'epistola di Canace si chiudeva su un'eroina ormai risoluta ad assecondare con amaro coraggio la violenza di un padre spietato, una giovane donna e madre la cui unica preghiera può essere ormai quella di una sepoltura comune con la sua sfortunata creatura:

Ov. Her.11, $97 \mathrm{~s}$.

scimus, et utemur violento fortiter ense; pectoribus condam dona paterna meis.

Ov. Her.11, 121-28

tu tamen, o frustra miserae sperate sorori, sparsa, precor, nati collige membra tui,

et refer ad matrem socioque inpone sepulcro, urnaque nos habeat quamlibet arta duos!

vive memor nostri, lacrimasque in vulnera funde, neve reformida corpus amantis amans. 
tu, rogo, dilectae nimium mandata sororis

perfice; mandatis obsequar ipsa patris!

\section{tragica di Canace che consegna infine Violante al gioco della Fortuna, la miracolosa dea} del romanzo.

\section{NOTE}

1. V. Branca, Boccaccio medievale e nuovi studi sul Decameron, Firenze $1997^{9}$.

2. Dizionario critico della letteratura italiana, Torino, 1974, pp. 351 ss. (= Boccaccio, Decameron, a cura di V. Branca, Torino 1999, pp. LIV ss.). Il problema delle 'fonti' della novella boccacciana è inquadrato in un'ottica metodologicamente più adeguata agli studi sull'intertestualità e impegnata a distinguere varie forme e funzioni del rapporto tra testi, modelli narrativi, generi in C. Di Girolamo-Ch. Lee, Fonti, in Lessico critico decameroniano, a cura di R. Bragantini- P. M. Forni, Torino 1995, pp. 142 ss.

3. Lucia Ricci Battaglia, Giovanni Boccaccio, in Storia della letteratura italiana, diretta da E. Malato, vol. II, Il Trecento parte II, Gli albori dell'umanesimo, Roma 1995, p. 818, la quale opportunamente ricorda un articolo di A. Limentani, Boccaccio e le sue fonti, in "Cultura e scuola" 8, 1963, pp. 5 ss. I materiali su cui si è esercitato il bricolage boccacciano sono raccolti e classificati in repertori che si sono costituiti a partire dalla stagione positivistica degli studi di letteratura: M. Landau, Die Quellen des Dekameron, Stuttgart 1884, A. C. Lee, The "Decameron". Its Sources and Analogues, London 1909; G. Gröber, Über die Quellen von Boccaccios Dekameron, Strasbourg 1913.

4. L. Rossi, Presenze ovidiane nel Decameron, in "Studi sul Boccaccio" 21, 1993, pp. 25-137; R. Hollander, The Proem of the Decameron: Boccaccio between Ovid and Dante, in Miscellanea di Studi Danteschi in memoria di Silvio Pasquazi, Napoli 1993, pp. 102-107.

5. Vedi soprattutto G. Rosati, Epistola elegiaca e lamento femminile, saggio intr. a P. Ov. Nasone, Lettere di eroine, a c. di G. R., Milano 1989, pp. 5-62.

6. G. Rosati, Epistola elegiaca cit., pp. 41 ss.

7. A. C. Lee, op. cit., p. 165.

8. Il riferimento è all'opera citata alla n. 3 .

9. Boccaccio, Decameron, a cura di V. Branca, cit. pp. 1293 s.

10. Su questo concetto vd. A. Barchiesi, Problemi d'interpretazione in Ovidio: continuità delle storie, continuazione dei testi, "MD" 16, 1986, pp. 77-107; Narratività e convenzione nelle Heroides, "MD" 19, 1987, pp. 63-90.

11. Per i problemi di ricostruzione vedi soprattutto H. Jacobson, Ovid's Heroides, Princeton 1974, pp. 159 ss. (con indicazione della precedente bibliografia); vd. anche M. Labate, La Canace ovidiana e l'Eolo di Euripide, “ASNSP" s. III, 7, 1977, pp. 583 ss.

12. Per una succinta discussione di singoli problemi esegetici, si vedano le note ad l. di Conington Nettleship e di Austin.

13. Dec. 5, 7, 5 "come che egli a guisa di servo trattato fosse nella casa pur co' figliuoli di messer Amerigo si crebbe".

14. Vedi i lavori citati sopra alla n. 11.

15. Ov. Her. 11, 21-24 O utinam, Macareu, quae nos commisit in unum,/venisset leto serior hora meo!/ cur umquam plus me, frater, quam frater amasti,/ et tibi, non debet quod soror esse, fui? 
16. Anche se poi, nella strategia narrativa della novella, questo tratto si rivelerà funzionale allo scioglimento fortunato della vicenda: proprio l'esitazione della vittima tra i due strumenti di morte rallenta il precipitare degli eventi e consente l'intervento salvifico della fortuna.

\section{RIASSUNTI}

Negli studi sul Decameron è consolidato un giudizio fortemente limitativo della rilevanza degli autori antichi, tra quelli che forniscono gli ipotesti delle singole novelle. Scopo di questo lavoro è mostrare, attraverso un esempio, come, nel quadro dei materiali letterari e narrativi che svolgono una funzione rilevante nel laboratorio dell'intertestualità decameroniana, la presenza della letteratura classica sia meno modesta di quanto sembrerebbe risultare dalla vulgata critica, e non si eserciti soltanto in maniera indiretta, o al livello di lontane matrici culturali o di archetipi di generi, di modalità o registri narrativi, ma possa anche proporsi come specifico rapporto tra testi. L'autore classico in questione è Ovidio, il testo una delle Heroides (precisamente la XI, Canace a Macareo), la novella in cui l'epistola ovidiana traspare in filigrana è Decameron V 7, a proposito della quale, secondo repertori e commenti, non sarebbero rintracciabili, al di là di singoli spunti e di un generico riferimento al tradizioni romanzesche, significative presenze di 'fonti' o 'modelli' riconoscibili. L'intertesto ovidiano, imponendosi sull'iniziale spunto romanzesco, orienta decisamente (anche se provvisoriamente) la storia dei due giovani amanti verso una cupa tragedia, ponendo al centro della novella il contrasto tra un padre autoritario e la sua fragile figlia, vinta da una passione d'amore che le circostanze hanno reso irresistibile. Quando ormai la falsariga del modello ovidiano sembrerebbe destinata a condurre i protagonisti verso un destino di morte, l'improvviso riaffiorare del tema romanzesco (il fanciullo rapito e il gioco della fortuna) conduce all'anagnorisis e a quell'insperato lieto fine che la regina ha imposto ai narratori come motivo-guida della giornata.

\section{INDICE}

Mots-clés : Boccaccio, Decameron, Heroides, intertestualità, Novella, Ovidio, Romanzo, Tragedia 